\title{
EVOLUCIÓN DE LA EVALUACIÓN EDUCATIVA EN EL CONTEXTO ECUATORIANO
}

Flores Obando Juan Marcelo. ${ }^{1}$, Placencia Camacho María del Carmen. ${ }^{2}$, Moya López Carlos Fernando. ${ }^{3}$ \& Quintanilla Vásquez María José. ${ }^{4}$

\begin{abstract}
This article analyzes the different historical moments that the educational evaluation has had in the Ecuadorian context. The objective is to comment on the evolution of the different forms of evaluation; from the time when the evaluation was established as a mechanism of punishment and persecution, and the changes and transformations that have arisen to date with an evaluation that is based on the quality standards promoted by the Ministry of Education of Ecuador.

In Ecuador, according to MINEDUC (2007) from 1996 to 2007, the tests (APRENDO) 2007 have been applied four times to the students of: third, seventh and tenth of Basic education in the areas of mathematics, language and communication ; in 2008, the SER ECUADOR tests were made official to students in the fourth, seventh and tenth grades of basic education and third year of high school in the areas of language, communication, mathematics, social studies and natural sciences; with the tests "Ser Estudiante" applied to the students of fourth grade of basic, tests "Ser Estudiante" 2010, tests "Ser Bachiller" 2017; which shows that the highest percentage of students do not reach a level of educational excellence and on the contrary many of the students are at the elementary level of academic performance.

In conclusion, the evaluation must fulfill the objective of orienting, guiding and reinforce learning as the end of the educational process that is to form the student in an integral way to achieve personal and professional satisfaction.
\end{abstract}

Keywords: Evaluation, Mechanism, Punishment, Persecution, Standards.

CÓDIGO UNESCO: Sistemas de Información 120318

\footnotetext{
${ }^{1}$ Unidad Educativa "Rumiñahui”, Ambato, Ecuador, jmflores_ob70@hotmail.com

2 Unidad Educativa "Mario Cobo Barona", Ambato, Ecuador, mplacencia12@gmail.com

${ }^{3}$ Unidad de Apoyo a la Inclusión de Shushufindi, Shushufindi, Ecuador, moya.lopez@gmail.com

${ }^{4}$ Unidad Educativa Especializada "Carlos Garbay", Riobamba, Ecuador, majito1588@outlook.com
} 


\section{RESUMEN}

El presente artículo analiza los diferentes momentos históricos que ha tenido la evaluación educativa en el contexto ecuatoriano. El objetivo es comentar la evolución que han tenido las distintas formas de evaluación; desde la época en que la evaluación era establecida como un mecanismo de castigo y persecución y trasformaciones surgidos hasta la actualidad con una evaluación que se fundamenta en los estándares de calidad promovidos por el Ministerio de Educación del Ecuador.

En el Ecuador según MINEDUC (2007) desde el año 1996 hasta el 2007 se han aplicado en cuatro ocasiones las pruebas (APRENDO) 2007, a los estudiantes de: tercero, séptimo y décimo de educación Básica en las áreas de matemática y lenguaje y comunicación; en el 2008 se oficializaron las pruebas SER ECUADOR, a los estudiantes de los grados: cuarto, séptimo y décimo de educación básica y tercero de bachillerato en las áreas de lenguaje y comunicación, matemática y se incluyó estudios sociales y ciencias naturales; con las pruebas Ser Estudiante aplicadas a los dicentes de cuarto grado de básica, pruebas ser estudiante 2010, pruebas ser bachiller 2017; las cuales demuestran que el mayor porcentaje de estudiantes no alcanzan un nivel de excelencia educativa y por el contrario muchos de los estudiantes se encuentran en el nivel elemental de rendimiento académico.

En conclusión la evaluación debe cumplir el objetivo de orientar, guiar y reforzar el aprendizaje como fin del proceso educativo que es formar integralmente al dicente para alcanzar la satisfacción personal y profesional.

Palabras Clave: Palabras clave: evaluación, mecanismo, castigo, persecución, estándares.

\section{INTRODUCCIÓN}

Desde el primer método sistemático de evaluación desarrollado por Ralph Tyler, hasta las pruebas APRENDO, PISA, y SER, desde el enfoque del castigo o premio hasta la evaluación como un proceso de diálogo, comprensión y mejora, así han sido los cambios y evoluciones que se han dado al pasar los años, el avance tecnológico, el cambio de matriz productiva, el progreso y desarrollo de centros educativos acorde a las necesidades del país son los ámbitos predominantes para la generación de una educación de calidad, la consecución de un mejor perfil de salida de estudiantes a nivel medio a superior y la formación permanente de profesionales, nos han guiado para poder concebir la idea correcta sobre lo que es en realidad la evaluación, tomando así a este proceso como una 
actividad indispensable y previa a toda operación, que antecede a las decisiones y a la acción humana.

El propósito de este presente artículo referativo sobre la Evolución de la Evaluación Educativa en el Contexto Ecuatoriano es abordar sobre la evolución cronológica de la evaluación educativa, a través de la historia en nuestro contexto ecuatoriano y por ello surge la interrogante: la cual contiene eventos sucesivos que resumen

¿Por qué es necesario investigar la evolución histórica que ha tenido la evaluación educativa en Ecuador?

Es por ello que hemos visto importante realizar el presente artículo, ya que, permitirá conocer cuál ha sido la evolución, su trascendencia, conocer sus falencias, sus mejoras, sus aciertos y debilidades de la evaluación a través de los años, evaluación de la sociedad, del contexto así como también del concepto de evaluación.

\section{DESARROLLO}

Al considerar el tema evolución de la evaluación educativa surgen dos partes diferenciadas a decir de (Elola, 2000) la primera de ellas consiste en una aproximación conceptual a la idea de evaluación y la segunda parte está integrada por un glosario que recoge los principales conceptos vinculados con los procesos de evaluación y los define de modo operativo.

Además (Ahumada, 2001) manifiesta que:

El concepto de evaluación ha ido evolucionando en consonancia con el concepto de educación predominante. Es así que desde una evaluación centrada en el acto de juzgar el valor de las cosas, se ha evolucionado hacia una evaluación que pretendía asignar valores precisos de medición a determinados objetos educativos.

Y luego (Ahumada, 2001) añade "la concepción de juicio predominó durante varios siglos y solo a fines del siglo XIX fue paulatinamente reemplazada por la concepción de "medición".

En la década de los 30, según (Ahumada, 2001) "Ralph Tyler inicia un movimiento de la evaluación en función del logro de determinados objetivos formulados con antelación, produciendo un cambio relevante en para poder comprender el porqué del proceso educativo, el cual siempre estaba enfocado a ver los resultados del aprendizaje".

Años más tarde (1950), según: (Morales, 2001) Tyler mismo es el encargado de señalar que en base a los objetivos planteados y los resultados obtenidos se puede conocer cuál es el grado de consecución, con ello se establece la programación basada en objetivos, donde por 
primera vez se puede establecer un concepto de evaluación refiriéndose como: " $E l$ progreso que permite determinar en qué grado han sido alcanzados os objetivos educativos propuestos."

Es así que en los años 60 el interés en y por la educación aumenta de una manera acelerada donde ya surge la educación permanente y global pero a la vez surte la preocupación e interés por el fracaso escolar, donde esto conlleva a tomar en cuenta tanto a la educación educativa como a la empresarial con la aparición y diseño de instrumentos de evaluación fiables y útiles. (Morales, 2001).

De esta manera (Morales, 2001) menciona que, según Cronbach(1963) la evaluación se ha de entender como una fase de "recogida y uso de información para tomar decisiones sobre un programa educativo" dejando así el concepto que se tenía de evaluación como un instrumento de control y medida con intención de llevar a cabo la valoración final de un proceso sino más bien como un medio que brinda la posibilidad de retroalimentar el proceso educativo y la toma de decisiones procesos que permitirán elevar el proceso educativo para un mejor alcance de objetivos.

En la década de los 70' se logra establecer una alteración en las ideas por la aparición de Piaget con su concepción constructivista del aprendizaje, donde surgen opciones cualitativas que priorizan el proceso y el uso de procedimientos antropológicos, así o manifiesta (Morales, 2001) donde se establecen diferentes ideas entre ellas "la evaluación iluminativa" donde según Parlett y Hamilton 1977 "el objetivo principal es la descripción y la interpretación, más que la valoración y la predicción, considerando así que la nueva propuesta evaluativa requiere no sólo del cambio de metodologías sino también de nuevas suposiciones y conceptos para comprenderla desde dos aspectos: el sistema de enseñanza y el medio de aprendizaje." es aquí que a partir de estas concepciones la exclusividad de aplicar la evaluación a los estudiantes y más bien se desarrollan otros elementos en los docentes, en centros y en los programas educativos.

Además (Ahumada, 2001) añade que actualmente se tiende a aceptar una concepción ecléctica del proceso de evaluación, manifestándose que la evaluación "es el proceso de delinear; obtener; procesar y proveer información valida, confiable y oportuna que nos permita juzgar el mérito o valía de programas, procedimientos y productos con el fin de tomar decisiones".

Para (Gutiérrez, 2011) como resultado de las políticas orientadas al mejoramiento cualitativo de las instituciones, de sus programas, de su trabajo docente se ha desatado una fiebre evaluadora a nivel de todos los estamentos de la educación y son muy pocas las instancias que han escapado a los efectos de esta evaluación.

En nuestro país actualmente se han adoptado normas de evaluación definidos como estándares de aprendizaje que, a decir de (Gutiérrez, 2011) el término estándar es muy 
usado en educación, pero su significado y propósito no están claros, es ¿una meta?, ¿una medida de progreso?, un patrón o norma? ¿una unidad común para comparar resultados?

Además (Gutiérrez, 2011) añade que en educación el término estándar tiene tres usos comunes:

1. Estándares de contenido o curriculares

2. Estándares de desempeño escolar

3. Estándares de oportunidad para aprender

Para (Flórez, 2007) el enfoque de evaluación para el aprendizaje se presenta en contra de las prácticas más habituales en evaluación que transforman en rutina esta actividad, ignoran los aprendizajes centrales de cada disciplina, disocian la evaluación de los procesos enseñanza-aprendizaje y entregan notas sin ninguna aclaración de su significado.

Para (Leyva, 2010) uno de los obstáculos que con mayor frecuencia tienen las instituciones educativas para realizar un trabajo sistemático de evaluación del aprendizaje consiste en la falta de una cultura de la evaluación entre los actores del proceso educativo - alumnos, profesores y directivos-, lo que conduce a prácticas evaluativas que no son congruentes con los contenidos, enfoques y propósitos del modelo educativo.

Según Ponce (2010), los encargados de elaborar políticas educativas implementaron un sistema de pruebas de logros académicos (APRENDO) en 1996. Sin embargo, el sistema no disponía de su propio presupuesto y se ejecutó hasta el año 2000. Adicionalmente, el sistema afrontó la resistencia del poderoso sindicato de profesores (UNE), que consideró que el sistema evaluaría el desempeño de los profesores de manera injusta.

Por otra parte Román \& Murillo (2008) consideran que los docentes son actores claves y relevantes para la calidad de los procesos de enseñanza y aprendizaje es algo de lo cual hay no sólo consenso social, sino que cada vez es más sólida la evidencia empírica que lo confirma. Las competencias profesionales de los profesores, principalmente referidas a la preparación de la enseñanza y a la organización del proceso de trabajo en el aula, se consolidan como factores importantes que inciden en lo que aprenden y logran los estudiantes. Desde allí, entonces, es que interesa conocer cuan preparados están dichos profesionales para implementar procesos de calidad, que asuman la diversidad de estudiantes que llegan a sus aulas con el anhelo y el derecho de aprender a ser y a desenvolverse exitosamente en la escuela y en la sociedad.

González ( 2002), defienden que un aspecto destacable a evaluar es la comprensión, por el estudiante, de la actividad a realizar, su significado y su sentido, su plenitud y la forma en que se accede a dicha comprensión, como contenido de la necesaria orientación que marca calidades diferentes en el aprendizaje. 
Según Ravela y otros (2008), la importancia de las evaluaciones nacionales de logros educativos es conocer mejor la dinámica de procesos y resultados en los sistemas educativos que es cada vez más frecuente a nivel regional y mundial, en países de muy diversas culturas y orientaciones ideológicas de gobierno, además se pretende a través de la aplicación de instrumentos de evaluación, medir tanto actitudes como aptitudes del estudiante para conocer el proceso, progreso y resultados del proceso educativo; es decir, se desea conocer cuáles han sido los conocimientos, habilidades, destrezas y valores desarrollados, como resultado del proceso educativo y su aplicación en la vida cotidiana.

Prueba de ello es la creciente participación de los países en las evaluaciones internacionales como PISA, TIMSS y PIRLS, y regionales como SERCE (en América Latina) y SACMEQ (en África), así como el desarrollo de diferentes tipos de sistemas nacionales y sub nacionales de evaluación.

En algunos casos, la evaluación está motivada por una preocupación por la formación ciudadana y la consolidación de una sociedad democrática; en otros, por la productividad de la fuerza de trabajo y la competitividad de la economía nacional. En casi todos los casos se asume que la evaluación puede servir:

- Como base para adoptar políticas educativas mejor fundamentadas;

- Para mejorar la gestión de los sistemas educativos;

- Como instrumento para la colaboración y el aprendizaje continuo al interior de los mismos.

- Para evaluar los factores intraescolares y extraescolares que inciden en el aprendizaje de los estudiantes.

Con relación a los estándares o criterios e indicadores para la evaluación de logros Machado (2000), sostiene que los estándares educacionales definen las competencias y habilidades básicas que deberían adquirir los niños y jóvenes durante su tránsito por el sistema escolar. Definen y comunican con claridad a distintas audiencias, no sólo las especializadas, lo que los estudiantes deberían saber en cada área de conocimientos en cada grado o nivel y lo que deberían saber hacer con esos conocimientos.

Se piensa que definir con claridad y elevar esos estándares contribuirá a mejorar los logros de aprendizaje de todos los alumnos, movilizando sus propios esfuerzos y los de todos los otros actores del sistema que inciden sobre sus logros.

A través de la historia ecuatoriana, desde 1830; se han realizado varias constituciones en nuestra república y poco se menciona en ellas sobre evaluación educativa, es a partir de 1996 hasta el año 2007 que se empezó a un proceso serio de evaluación, con la aplicación de las pruebas APRENDO en el sistema escolarizado en diferentes áreas y de manera muestral; luego de ello en la Constitución de Montecristi de octubre del 2008 se señala en 
el artículo 346 que existirá una institución pública con autonomía de evaluación integral interna y externa que promueva la calidad de la educación, dicho precepto se lo logra con la reforma a la Ley Orgánica de Educación Intercultural y Bilingüe que en su artículo 67 dispone de la creación del Instituto Nacional de Evaluación Educativa hoy conocido como (INEVAL) y de igual manera se aplican las pruebas Ecuador.

Es así que llegamos a la actualidad donde por todas estas razones se pretende aplicar la evaluación en base a los estándares de: gestión educativa, de desempeño profesional, de aprendizaje, y de infraestructura, ya que su principal propósito es orientar, apoyar y monitorear, la acción de los diversos actores del sistema educativo para una formación continua, el alcance del éxito, y sobre todo para la mejora y consecución de la calidad educativa. (Ministerio de Educación, 2012).

Así empezaremos con la importancia de la evaluación de la gestión educativa la cual nos permite conocer la organización y desarrollo de procesos de gestión tanto de la institución como el desarrollo profesional de os docentes, todo esto vinculado al funcionamiento óptimo y a la mejora de la calidad de aprendizajes, con esto se pretende que los agentes involucrados en el proceso educativo sean capaces de tomar decisiones, comunicarse, manejar y enfrentar conflictos, trabajar en equipo, con y entre los miembros de la comunidad de acuerdo a la realidad propia de cada institución lo cual accederá en base a resultados elaborar planes, instrumentos, adoptar metodología o técnicas que den paso a establecer planes de mejora necesarios para el alcance de la calidad. (Ministerio de Educación, 2012)

En cambio los estándares de Desempeño Profesional son los que orienta a mejorar la labor profesional tanto de docentes como de directivos involucrados en el sistema educativo, estos estándares ayudan a guiar, evaluar, autoevaluar, diseñar, y tomar decisiones en lo que refiere a Evaluación, Certificación, Méritos y Oposiciones, Formaciones: inicial y continua y sobre todo para obtener mejores resultados en el desarrollo profesional educativo, para en conjunto con los otros aspectos alcanzar la calidad en base a la evaluación de varios factores que permitirán la permanencia, cambio y evolución. (Ministerio de Educación, 2012)

Por otro lado los Estándares de Aprendizaje simplemente son los logros que los estudiantes deben alcanzar en todo el trayecto escolar, desde el primer año de Educación Básica hasta el tercer curso de Bachillerato, medidos y evaluados en cinco niveles establecidos en las cuatro áreas básicas: Lengua y Literatura, Matemática, Estudios Sociales, Ciencias Naturales. 
Tabla N1 Niveles de escolaridad

NIVELES

Primer nivel

Segundo nivel

Tercer nivel

Cuarto nivel

Quinto nivel

\section{AÑ̃S BÁSICOS}

Al término de Primer año de E.G.B

Al término de cuarto año de E.G.B

Al término de séptimo año de E.G.B

Al término de Décimo año de E.G.B

Al término de Tercer año de Bachillerato.

Fuente: (Ministerio de Educación, 2012)

Y así llegamos al último estándar que es el de Infraestructura, que son los criterios para la construcción y la distribución de los espacios escolares, estos indican las condiciones arquitectónicas que deben poseer las instituciones los mismos que buscan la satisfacción de requerimientos pedagógicos para el mejoramientos de la calidad en la educación y el alcance de niveles óptimos en el proceso enseñanza aprendizaje. (Ministerio de Educación, 2012)

Ilustración N1 Infraestructura

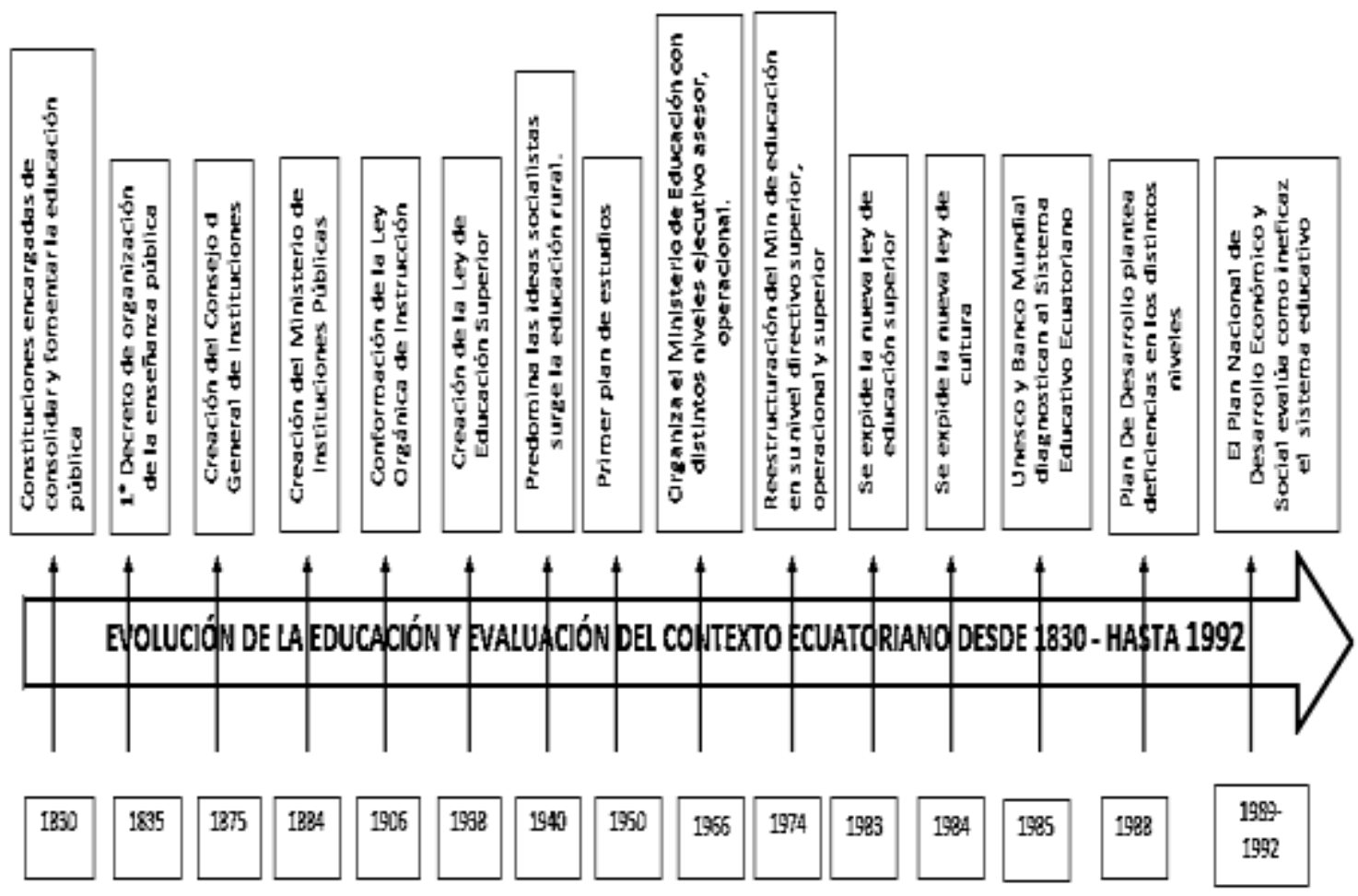

Fuente autor: Grupo Investigativo 
Ilustración N2 Evolución de la Educación

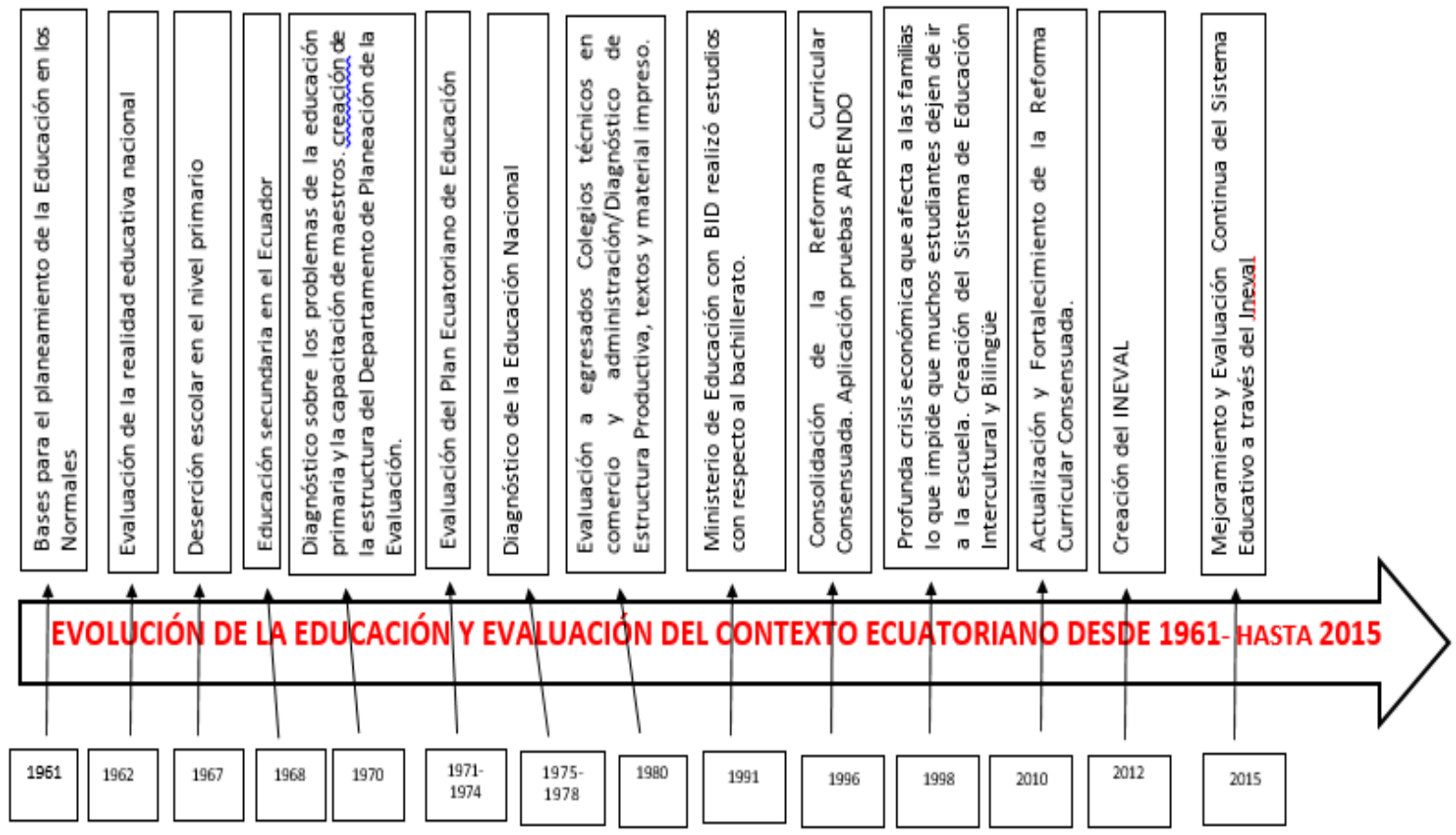

Fuente autor: Grupo Investigativo

\section{CONCLUSIONES}

- La práctica evaluativa en el contexto ecuatoriano ha tenido varias transiciones por lo tanto se concluye que cada proceso terminado ha servido como base para impulsar un mejor sistema educativo de calidad.

- Cada una de las políticas educativas impulsadas por las autoridades de turno han motivado y dado prioridad a la educación en el contexto ecuatoriano.

- Los sistemas de evaluación educativos actuales apuntan a la calidad irremediable de la gestión, aprendizajes, infraestructura y desempeño docente, por lo que se concluye que de continuar con cada uno de los indicadores planteados sin duda alguna en pocos años se observarán avances en cada uno de los ámbitos de desarrollo.

- Los actores involucrados en la educación debemos comprometernos con el cambio sistemático evolutivo sobre la evaluación con mentalidad transformadora para alcanzar la formación de una sociedad cada vez mejor.

RECEIVED: April, 2017

REVISED: August, 2017 


\section{Referencias bibliográficas}

Ahumada, P. (2001). La evaluación en una concepción de aprendizaje significativo. Valparaiso: Edisiones universitarias de Valparaíso.

Elola, N. (2000). Evaluación educativa: una aproximación conceptual. Buenos Aires: Ediciones Educativas.

Flórez, M. T. (26 de ENERO de 2007). Educar Chile. Obtenido de http://www.educarchile.cl/ech/pro/app/detalle?id=190202

González Pérez, M. ( 2002). La evaluación del aprendizaje. CEPES Universidad de la Havana, 9.

Gutiérrez, H. (2011). La nueva evalución educativa. Desempeños, logros, competencias y estándares. Chile: Magisterio.

Leyva, Y. (1 de Marzo de 2010). Recuperado el 11 de Noviembre de 2015, de Unam: http://www.ses.unam.mx/curso2012/pdf/Guia_evaluacion_aprendizaje2010.pdf

Machado, A. (2000). Análisis de prospectivas de la educación en la región de América Latina y el Caribe. Santiago de Chile: Andros.

Ministerio de Educación. (2012). Estandares de Calidad Educativa, Aprendizaje, Gestión Escolar, Desempeño e Infraestructura. Recuperado el 12 de Noviembre de 2015, de http://educacion.gob.ec/wpcontent/uploads/downloads/2012/09/estandares_2012.pdf

Morales, J. (2001). La Evaluación en el Área de Educación Visual y Plastica en la ESO. 2001, 168. Barcelona, España.

Ponce, J. (2010). Políticas educativas y desempeño una evaluación de impacto de programas educativos focalizados en el Ecuador. Quito: ATRIO.

Ravela, P., Arregui, P., Valverde, G., Wolfe, R., Ferrer, G., Martínez Rizo, F., . . Wolff, L. (2008). Las evaluaciones educativas que America Latina necesita. Revista Iberoamericana de Evaluación Educativa, 1(1).

Román, M., \& Murillo, J. (2008). La evaluación del desempeño docente: objeto de disputa y fuente de oportunidades en el campo educativo. Revista Iberoamericana de Evaluación Educativa, 1(2), 2. 


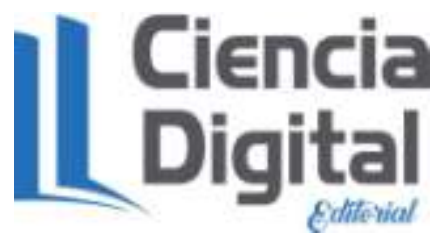

El artículo que se publica es de exclusiva responsabilidad de los autores y no necesariamente reflejan el pensamiento de la Revista Ciencia Digital.

El articulo queda en propiedad de la revista y, por tanto, su publicación parcial y/o total en otro medio tiene que ser autorizado por el director de la Revista Ciencia
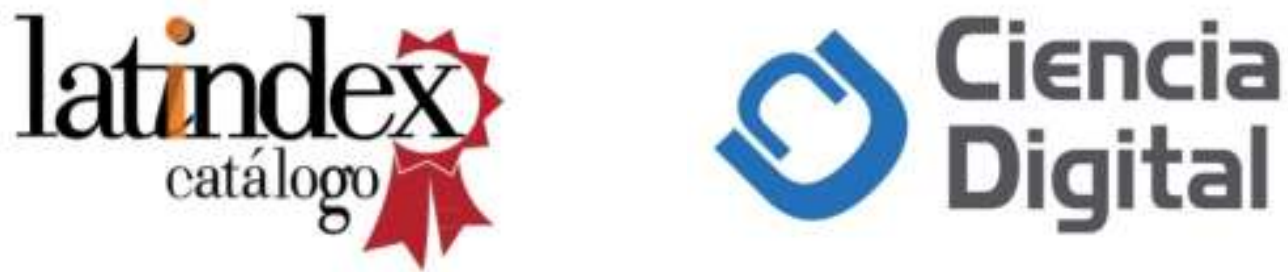